\title{
Computer-Guided Concentration-Controlled Trials in Autoimmune Disorders
}

\author{
*J. McMichael. ${ }^{*}+R$. Lieberman, ${ }^{*}$ H. Doyle, ${ }^{*}$ J. McCauley, $\ddagger D$. Van Thiel, \\ *A. Thomson, *J. Fung, and *T. E. Starzl \\ *Transplantation Institute. University of Pittsburgh, Pittsburgh, Pennsylvania; + Staff College, Center for Drug \\ Evaluation and Research, Food and Drug Administration, Rockville, Maryland: and Oklahoma Transplant Institute, \\ Oklahoma City, Oklahoma, U.S.A.
}

\begin{abstract}
Summary: A randomized concentration-controlled clinical trial (RCCCT) is an alternate experimental design to the standard dose-controlled study. In a RCCCT. patients are randomly assigned to predefined plasma or blood drug concentration ranges (low, medium, and high). With the caveat that concentration ranges are sufficiently separated, this design should enhance the ability to discover important concentration response relationships. FK-506, a potent and promising immunosuppressive agent for prevention and treatment of graft rejection. has shown significant clinical activity in some immune-mediated disorders. To implement the RCCCT design, a novel FK-506 intelligent dosing system (IDS) was used to guide all doses to prospectively achieve the target concentration range specified in the study protocol. Patients enrolled in these trials suffered from a variety of autoimmune disorders, including multiple sclerosis, primary biliary cirthosis, psoriasis, autoimmune chronic active hepatitis, and nephrotic syndrome. We observed excellent predictive performance of the IDS for all patients. The accuracy (mean prediction error) of the IDS was $-0.022 \mathrm{ng} / \mathrm{ml}$ and the precision (standard deviation of the prediction error) was $0.119 \mathrm{ng} / \mathrm{ml}$. Thus, the IDS is both accurate and reproducible for autoimmune patients. We conclude that the RCCCT design, guided by an accurate and precise IDS, is an informative and cost-effective approach for evaluation of efficacy and safety of effective but highly toxic agents. Key Words: Concentration-controlled artificial intelligence-Dynamic dosing-Autoimmune diseases.
\end{abstract}

In late 1991. we began randomized concentrationcontrolled clinical trials (RCCCTs) designed to evaluate the efficacy and toxicity of FK-506 in certain autoimmune diseases being studied at the University of Pittsburgh Medical Center (UPMC) (Table 1). Concentration-controlled trials (CCTs) were considered the best choice for evaluating FK-506 because of the narrow therapeutic index of FK-506

Address correspondence and repnnt requests to $\mathrm{Mr}$. Juhn McMichael at University of Pittsburgh, 3601 Fifth Avenue. 5C Falk Clinic. Pittsburgh. PA 15213. L'.S.A. as well as the considerable sample size advantages that CCTs offer over standard trial designs (1). Substantially smaller sample sizes can be used when conducting CCTs since CCTs, by design, minimize interindividual drug level variability within comparison groups and, consequently, decrease withingroup clinical variability. This design will facilitate carly assessment of efficacy, while providing information on concentration (dose) response. Another reason we found CCTs very attractive was that this pilot trial design does not necessitate a placebo group, which we believed would be unethical for many of the diseases we wished to study. 
TABLE 1. Autoimmune diseases

Rheumatology
Systemic sclerosis
Polymyositis dermatomyositis
Rheumatoid arthritis
Systemic lupus
Systemic vasculitides
Behçet's disease
Ophthalmology
Uveitis
Episcleritis
Retinitis
Gastroenterology
Autoimmune chronic active hepatitis
Inflammatory bowel disease
Primary biliary cirrhosis
Primary sclerosing cholangitis
Sprue
Neurology
Multiple sclerosis
Chronic inflammatory demyelinating polyneuropathy
Autoimmune inner ear disease
Endocrinology
Type I diabetes mellitus
Nephrology
Nephrotic syndrome
Glomerulonephritis
Dermatology
Alopecia areata totalis and universalis
Pyoderma gangrenosum
Severe psoriasis
Epidermolysis bullosa

\section{METHODS}

Approximately 300 patients meeting the specific entry criteria for the protocol in which they were enrolled were randomized to either a high. medium. or low FK-506 plasma concentration range using a block randomization design for three groups. This allocation design ensures that an equal number of patients will be in any one of the treatment groups at any point in time. Randomization was carried out by sequential draw of assignment. and the three block sizes used were selected randomly with equal probability. The order of assignment within a block was determined by generating a random number between 0 and 1 and then rearranging the random numbers in ascending order. For these studies. the low average plasma concentration of FK-506 was targeted at $0.2 \mathrm{ng} / \mathrm{ml}$. with a range from 0.1 to 0.4 $\mathrm{ng} / \mathrm{ml}$. The intermediate average concentration was targeted at $0.6 \mathrm{ng} / \mathrm{ml}$, with a range from 0.4 to 0.8 . and the high average plasma concentration target was $1.0 \mathrm{ng} / \mathrm{ml}$. with a range from 0.8 to $1.2 \mathrm{ng} / \mathrm{ml}$. This approach substitutes for a preconceived $\mathrm{mg} /$ $\mathrm{kg} /$ day dosing scheme. which may he completely inappropriate for a given patient (Fig. 1). The starting oral dose regimen of FK-506 was usually 0.075 $\mathrm{mg} / \mathrm{kg} /$ day in divided doses given every $12 \mathrm{~h}$. In the interest of patient safety and compliance, all patients enrolled in these studies were prerandomized, kept in the low FK-506 plasma concentration range of $0.1-0.4 \mathrm{ng} / \mathrm{ml}$ until they were stable, and then moved to the zone to which they had been randomized. Failure of a patient to achieve or be maintained within his/her randomized zone was considered a treatment failure. To determine the FK-506 dosage required to achieve the desired target plasma concentration. an artificial intelligence dosing system (IDS), which would predict drug dosages and concentrations. was developed. To use this dosing system. the physician enters the patient's current FK-506 dose (mg/day), the patient's current plasma concentration $(\mathrm{ng} / \mathrm{ml})$, and the target plasma concentration $(\mathrm{ng} / \mathrm{ml})$. The IDS then calculates the FK-506 dose required to achieve target concentration.

In a CCT, patients are assigned to predetermined average plasma drug concentration ranges using an individualized dosing scheme. Simple linear regression is used to test the null hypothesis that the mean change in efficacy and toxicity parameters (for continuous variables) is not linearly related to FK-506 target plasma concentration. The $x^{2}$ test of association was used to test whether there is an association between the rate of nephrotoxicity at predefined time points and FK-506 plasma concentration.

Criteria for reducing a patient's FK-506 blood plasma concentration range included increase in the creatinine concentration of $150 \%$ above baseline. reduction in the creatinine clearance to $<40 \mathrm{cc} / \mathrm{min}$. severe neurotoxicity (as defined by tremors, sleep disturbances and seizures). and development of new onset insulin-dependent diabetes.

It was left to the discretion of the treating physi-

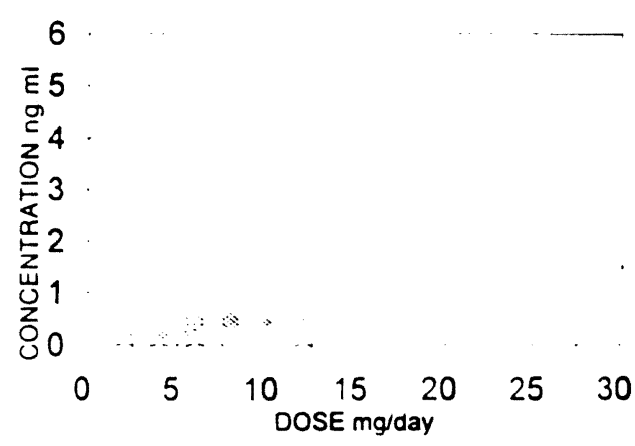

FIG. 1. Autoimmune disease pattents: starting dose versus concentration. The starting daily dose of FK -506 given appears to be unrelated to the resulting concentration. 
cian whether a patient should remain in his/her randomized zone. should that concentration of FK-506 not appear to be influencing the patient's disease state. If this occurred, the patient would be moved to the next zone and treated; however, he/she would be recorded as a treatment failure at the original zone to which he/she was randomized (intent to treat analysis).

FK-506 concentration in plasma was measured by enzyme-linked immunoabsorbent assay (ELISA), using a monoclonal antibody as previously described $(2,3)$. The coefficient of variation of this assay was $17 \%$ at $1.4 \mathrm{ng} / \mathrm{ml}, 14.4 \%$ at $2.9 \mathrm{ng} / \mathrm{ml}$. and $12 \%$ at $5.7 \mathrm{ng} / \mathrm{ml}$. The effective therapeutic range for FK-506 was estimated to be $0.5-2.0 \mathrm{ng} / \mathrm{ml}$ in transplant patients. and is currently still being defined in patients with autoimmune disorders. The UPMC currently measures FK-506 in both plasma and whole blood, and predictive models have been developed for both matrices. Fujisawa is still determining which matrix is best for monitoring FK-506. (Fujisawa Corporation. Deerfield. IL. unpublished data).

\section{RESULTS}

To validate our IDS, a prospective study was conducted at UPMC (4). Our patient population consisted of 32 adult liver $(n=17)$ and kidney $(n=$ 15) transplant patients. Predictions of patient plasma concentrations were made throughout their clinical courses. Performance of the IDS was assessed using a standard prediction analysis. The mean prediction error (MPE), which is calculated as the mean difference between the observed and the predicted target values. was calculated to be 0.016 $\mathrm{ng} / \mathrm{ml}$. The root mean squared prediction error (RMSPE), which is calculated as the standard deviation of the prediction errors. was $0.189 \mathrm{ng} / \mathrm{ml}$. The $95 \%$ contidence interval $(\mathrm{Cl})$ for the mean prediction error was calculated to be between -0.084 and 0.052 . This brackets our calculated accuracy (including zero), indicating there is little or no bias in the system's dosing predictions.

In 50 predictions of concentrations made in the autoimmune disease patient group. the MPE was calculated to be $-0.022 \mathrm{ng} / \mathrm{ml}$, the RMSPE was calculated to be $0.119 \mathrm{ng} / \mathrm{ml}$. and the $95 \% \mathrm{CI}$ was calculated to be between 0.011 and -0.055 (Fig. 2). These results. as tabulated in Table 2 . demonstrate the accuracy and precision of this IDS and the util-

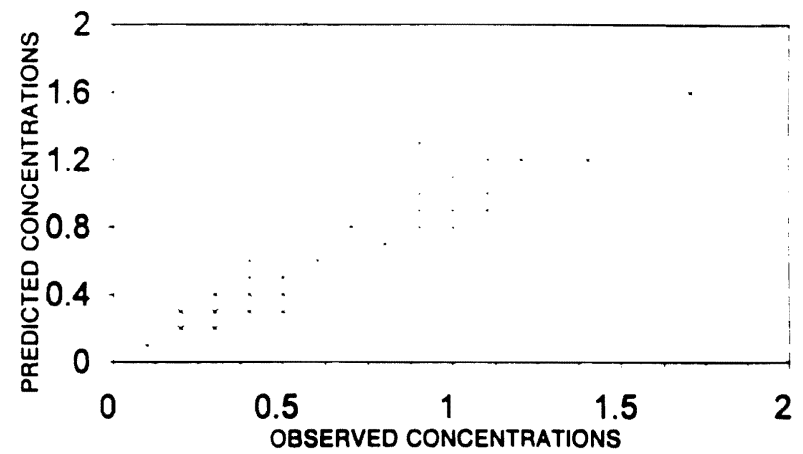

FIG. 2. Autoimmune disease patients: observed versus predicted FK-506 concentrations. The close correlation between the observed and predicted concentrations using the IDS.

ity of the system to move a patient to a desired concentration level and maintain that level.

\section{DISCUSSION}

The use of CCTs has been very effective in our early pilot studies to assess the efficacy and safety of FK-506 in autoimmune disorders. Using this study design, our final analysis will provide information about the toxic properties of FK-506 as it relates to plasma concentration. We will also have a relatively rapid. clear picture of the efficacy of FK506 in each of the autoimmune diseases studied and whether there is a relationship to plasma concentration. The use of the IDS has been very effective in providing physicians a simple method by which study patients can be taken to a desired plasma concentration and held there. This will simplify our analysis by providing clearly defined groups of study patients where statistical significance can be ascertained for any of the parameters being examined. Because using this IDS does not require any previous computer experience and it is user friendly, it will help minimize the learning curve associated with dosing new agents. e.g., FK-506. with its large pharmacokinetic variability and narrow therapeutic index. The IDS is ideally suited for multicenter trials since it would standardize therapy and aid in producing reliable homogenous results. independent of the study site.

TABLE 2. Autoimmune dise'ase patients' zone statistics

\begin{tabular}{lccc}
\hline Zone & $\begin{array}{c}\text { Mean } \\
\text { concentration }\end{array}$ & SD & No. \\
\hline Low & 0.27 & 0.12 & 10 \\
Medium & 0.66 & 0.16 & 10 \\
High & 1.08 & 0.19 & 10 \\
\hline
\end{tabular}




\section{REFERENCES}

1. Sanathanan LP. Peck CC. Temple R. Lieberman R. Pledger G. Randomization. PK-controlled dosing and titration: An integrated approach for designing clinical trials. Drug Information Assoc $J$ 1991:25:425-31.

2. Venkararamanan R. Jain A. Warty V, et al. Pharmacokinet- ics of FK506 in transplant patients. Trans Proc 1991;23: 2736-40.

3. Jain A. Abu-Elmagd K. Abdallah $\mathrm{H}$, et al. Pharmacokinetics of FK506 in liver transplant recipients following continuous intravenous infusion. J Clin Pharm (in press).

4. McMichael J. Irish W. McCauley J, et al. Evaluation of a novel "Intelligent" dosing system for optimizing FKS06 therapy. Trans Proc 1991:23:2780-2. 